\title{
Micro Damage and Cracking in Fibre Reinforced Composites by a Novel Hybrid Numerical Technique
}

\author{
Marco Lo Cascio, ${ }^{1,2, a)}$ Ivano Benedetti, ${ }^{1, \text { b) }}$ and Vladislav Mantič2, c) \\ ${ }^{1)}$ Department of Engineering, University of Palermo, Viale delle Scienze, Edificio 8, 90128, Palermo, Italy \\ ${ }^{2)}$ Escuela Técnica Superior de Ingeniería, Universidad de Sevilla, Camino de los Descubrimienos s/n, 41092 Sevilla, Spain \\ a) Electronic mail: marco.locascio01@unipa.it \\ b) Corresponding author: ivano.benedetti@unipa.it \\ ${ }^{c)}$ Electronic mail: mantic@us.es
}

\begin{abstract}
The prediction of failure mechanisms in fibre-reinforced composite materials is of great importance for the design of composite engineering applications. With the aim of providing a tool able to predict and explain the initiation and propagation of damage in unidirectional fiber reinforced composites, in this contribution we develop a micromechanical numerical model based on a novel hybrid approach coupling the virtual element method (VEM) and the boundary element method (BEM). The BEM is a popular numerical technique, efficient and accurate, which has been successfully applied to interfacial fracture mechanics problems of fibre-reinforced composite materials. The VEM has recently emerged as a powerful and robust technique, capable of dealing with very general polygonal/polyhedral meshes, including very distorted mesh elements, and just very recently it has also been applied to fracture and damage problems. In the present model, the BEM is used to model the fiber inclusions, which are not supposed to develop non-linear deformations or damage/crack in their interior, while the VEM, which generalizes the features of FEM, is used to model the surrounding matrix material, which can develop more complex behaviors. The implemented technique has been applied to a simple fracture problem and some promising preliminary results are shown and discussed.
\end{abstract}

\section{INTRODUCTION}

In the last few decades, thanks to remarkable developments in materials experimental characterization and to the advancements in high performance computing, a wealth of modelling strategies have been developed and implemented with the aim of simulating complex behaviours of materials and structures at different scales, embodying deeper and richer layers of fidelity. On the basis of such technological and modelling progresses, the multi-scale materials modelling and materials by design paradigms have gained noticeable benefits [1].

One of the aspects of relevant interest in the analysis and development of new materials for engineering applications is the understanding and possibly prediction of their degradation and failure mechanisms. In a multi-scale perspective, damage initiates at the micro-structural level, propagates and coalesces and eventually emerges at higher scales, where it can eventually affect the functionality or even the worthiness of structures [2]. The ability to predict damage initiation and evolution is crucial in several sectors: in aerospace applications, for example, a deeper understanding of damage initiation and propagation mechanisms is essential for the implementation of effective damage tolerance approaches and may have immediate repercussions on the planning of maintenance schedules, with direct implications in terms of costs.

In such a context, the ability to simulate with acceptable fidelity the micro-structure of materials and the complex non-linear interactions between its building blocks plays a fundamental role. On the other hand, the inclusion of deeper layers of fidelity requires the ability to robustly address several kinds of modelling complexities, included those arising, for example, from the need of representing involved material morphological details. In this respect, the development of computational techniques able to deal with complex and evolving geometries and meshes effectively and accurately attracts relevant interest.

In the present work, we propose a hybrid technique based on the simultaneous use of the Boundary Element Method (BEM) and the Virtual Element Method (VEM) for the analysis of crack propagation in fibre reinforced composite materials. The BEM is a numerical technique based on the use of boundary integral equations for the representation of the considered problems: its hallmark is a certain reduction in the dimensionality of the problem, direct consequence of the underlying integral formulation, with ensuing reduction in the number of degrees of freedom required in the analysis, with respect to other popular numerical techniques. It has been successfully employed for the analysis of solids and structures [3] and, more recently, in multi-scale materials modelling [4, 5, 6, 7, 8, 9]. The VEM is a recent and rapidly emerging generalisation of the Finite Element Method (FEM) which allows to include, without compromising the analysis accuracy, elements of very general shape, including polygonal elements with arbitrary numbers of

Fracture and Damage Mechanics

AIP Conf. Proc. 2309, 020001-1-020001-9; https://doi.org/10.1063/5.0033974

Published by AIP Publishing. 978-0-7354-4045-6/\$30.00 


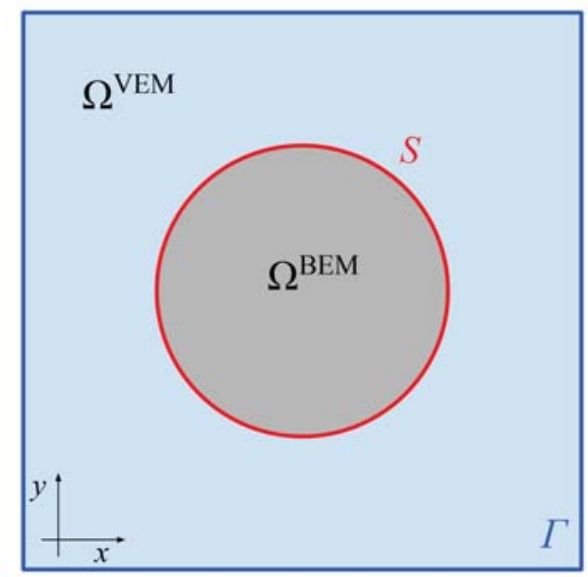

FIGURE 1: Geometry of the analysis domain: the BEM is employed to model the inclusion while the VEM models the surrounding matrix.

edges, non-convex elements and elements with curved edges $[10,11,12]$; the method has been successfully applied to materials homogenisation problems $[13,14]$.

The conjoined use of the BEM and VEM might provide some benefits in the analysis of complex micro-structures. Here the BEM is used to model the fibers, which are not expected to develop non-linear behaviours, while the VEM is employed to model the matrix, which may experience crack initiation and propagation. The inherent flexibility of the VEM with respect to the admissible elements shapes is fully exploited to avoid mesh-dependency in the crack propagation modelling, through a mesh topology modification restricted only to the elements containing the crack tip node. Such hybrid usage should in principle provide a reduction of the cost of the analysis and flexibility in the study of the matrix cracking. In this work we present some preliminary results and establish a workflow for future in-depth investigations.

\section{FORMULATION}

The multi-region two-dimensional domain $\Omega \subset \mathfrak{R}^{2}$ with external boundary $\Gamma=\partial \Omega$, as shown in Fig. 1, is considered in this study. It is assumed that no body forces act within $\Omega$, but either displacements or tractions can be enforced on the boundary $\Gamma$. The problem domain $\Omega$ is the union of two sub-domains, namely $\Omega^{\mathrm{BEM}}$ and $\Omega^{\mathrm{VEM}}$, which represent, respectively, the transverse section of a fibre and the the surrounding polymer matrix in a polymer fibre-reinforced composite. The two sub-domains share the interface $S$.

In this work, small strains and linear elastic material behaviour are assumed. As mentioned, the BEM is used to model the fiber inclusion while the VEM is used to model the surrounding matrix. For this purpose, $\Omega^{\mathrm{VEM}}$ is partitioned into a number of polygons of general shape, while the boundary $S=\partial \Omega^{\mathrm{BEM}}$ is divided into a number of straight segments, which form the edges of the polygonal elements in $\Omega^{\mathrm{VEM}}$ lying in proximity of the interface between the two sub-domains.

\section{Boundary Element modelling of the inclusion}

For the sub-domain $\Omega^{\mathrm{BEM}}$ with smooth boundary $S=\partial \Omega^{\mathrm{BEM}}$, if no body forces are applied, the boundary integral equation (BIE) for the displacements components $u_{j}$ at a boundary collocation point $\mathbf{x}_{0} \in S$ can be written, using tensor notation with $i, j=x, y$, as [15]

$$
\alpha_{i j}\left(\mathbf{x}_{0}\right) u_{j}\left(\mathbf{x}_{0}\right)=\int_{S} G_{i j}\left(\mathbf{x}_{0}, \mathbf{x}\right) t_{j}(\mathbf{x}) d s-\int_{S} H_{i j}\left(\mathbf{x}_{0}, \mathbf{x}\right) u_{j}(\mathbf{x}) d s,
$$


where $u_{i}(\mathbf{x})$ and $t_{i}(\mathbf{x})$ are the displacement and traction components at the integration boundary point $\mathbf{x}, G_{i j}\left(\mathbf{x}_{0}, \mathbf{x}\right)$ and $H_{i j}\left(\mathbf{x}_{0}, \mathbf{x}\right)$, respectively, are the components of the two-dimensional elasto-static fundamental solution for displacements and tractions under plane strain assumptions, and $\alpha_{i j}$ are the components of the free-term coefficient depending on the boundary geometry at the point $\mathbf{x}_{0}$.

The numerical treatment of the boundary integral formulation expressed by Eq. (1) is based on the subdivision of the boundary $S$ into a collection of $m$ straight elements $\Delta S_{k}$ and a convenient approximation of the boundary displacement and traction components in terms of shape functions and nodal values. In this work, a linear approximation of displacements and tractions over each boundary element is assumed, so to ensure a natural treatment of the interface conditions between the boundary element and the lowest-order VEM elements used to model the contiguous matrix. Using the approximations

$$
\mathbf{u}(\xi)=\mathbf{N}(\xi) \mathbf{u}^{k}, \quad \mathbf{t}(\xi)=\mathbf{N}(\xi) \mathbf{t}^{k}
$$

where $\mathbf{N}(\xi) \in \mathbb{R}^{2 \times 4}$ is the matrix collecting 1D linear shape functions for the boundary segment $\Delta S_{k}$, expressed as function of the natural coordinate $\xi$, and $\mathbf{u}^{k}, \mathbf{t}^{k} \in \mathbb{R}^{4 \times 1}$ collect, respectively, nodal components of displacements and tractions associated with the boundary segment $\Delta S_{k}$, Eq. (1) can be numerically integrated using the procedures described, e.g., in Ref. [3]. Such numerical integration, repeated for Eq. (1) written for the set of all the collocation points chosen along the boundary $S$, leads eventually to a global system of linear algebraic equations of the form

$$
\mathbf{H} \mathbf{U}^{\mathrm{B}}=\mathbf{G} \mathbf{T}^{\mathrm{B}}
$$

where the vectors $\mathbf{U}^{\mathrm{B}}, \mathbf{T}^{\mathrm{B}} \in \mathbb{R}^{2 m \times 1}$ collect the components of displacements and tractions of all collocation nodes identified along the boundary $S$ and $\mathbf{H}, \mathbf{G} \in \mathbb{R}^{2 m \times 2 m}$ collect the coefficients obtained from the numerical integration of Eq. (1) associated to such collocation points. It is worth noting that, when the BEM domain identifies an inclusion in the analysed domain, both $\mathbf{U}^{\mathrm{B}}$ and $\mathbf{T}^{\mathrm{B}}$ are unknown quantities that must be determined from the analysis.

\section{Virtual Element modelling of the matrix}

In this contribution, the lowest-order VEM formulation for two-dimensional linear elastic problems is employed to model the domain $\Omega^{\mathrm{VEM}}$, which is here associated with the polymer matrix of a fibre-reinforced composite material. Such a choice is motivated by the ensuing natural coupling with the linear boundary elements employed to model the fibre domain.

In the case of the lowest order VEM, for a general polygonal element $E$, the element degrees of freedom are the values of the displacements components at each of its $n$ vertex, which are collected into the vector $\mathbf{u}_{E}$. Analogously to the standard FEM, it is assumed that the displacements field $\mathbf{u}$ within the element can be expressed as

$$
\mathbf{u}=\mathbf{N}(\xi, \eta) \mathbf{u}_{E}
$$

where $\mathbf{N}(\xi, \eta)$ is the generic matrix containing the virtual shape functions $N_{v}(\xi, \eta)$ associated with each vertex $v$. Differently from standard FEM however, such shape functions are known only on the element edges of $E$, where they are globally continuous linear polynomials.

Since the shape functions $N_{v}(\xi, \eta)$ are not explicitly known within the polygonal element, from which their connotation as virtual, an explicit expression for the strains is not available. An approximated constant strain field $\varepsilon^{0}$ is assumed within each element, which can be computed from the degrees of freedom $\mathbf{u}_{E}$ as

$$
\varepsilon^{0}=\Pi_{E} \mathbf{u}_{E}
$$

where $\Pi_{E} \in \mathbb{R}^{3 \times 2 n}$ is the matrix representation of a projection operator defined as [11]

$$
\boldsymbol{\Pi}_{E}=\frac{1}{A_{E}} \sum_{v=1}^{n} \int_{e_{v}} \mathbf{N}_{v}^{E} \mathbf{N}(\boldsymbol{\eta}) d s
$$

where $A_{E}$ is the area of the polygonal element $E$, bounded by its $n$ edges $e_{v}$ and

$$
\mathbf{N}_{v}^{E}=\left[\begin{array}{cc}
n_{x} & 0 \\
0 & n_{y} \\
n_{y} & n_{x}
\end{array}\right]
$$


is the matrix containing the components $n_{x}$ and $n_{y}$ of the outward unit normal vector over each edge. The integrals appearing at the right-hand side of Eq. (6), which are carried out over the element edges, are exactly computable, since the restriction of the virtual shape functions $N_{v}$ to such edges are known piecewise linear polynomials.

The VEM elemental stiffness matrix $\mathbf{K}_{E}$ is given by the sum of two terms

$$
\mathbf{K}_{E}=\mathbf{K}_{E}^{c}+\mathbf{K}_{E}^{s}
$$

The consistency contribution is given by

$$
\mathbf{K}_{E}^{c}=A_{E} \boldsymbol{\Pi}_{E}^{\mathrm{T}} \mathbf{C} \boldsymbol{\Pi}_{E},
$$

where $\mathbf{C}$ is the material stiffness tensor in Voigt notation. On the other hand, $\mathbf{K}_{E}^{s}$ is a stabilisation term that may be computed as described, e.g., in Ref. [10]. Its presence is motivated by the need to avoid zero-energy modes not associated with rigid body motion, which may arise as a consequence of the fact that the approximate strains $\varepsilon^{0}$ are assumed constant within the element, while the displacements $\mathbf{u}$ are piecewise linear on the element edges, which in general may induce incompatibility between $\varepsilon^{0}$ and the nodal degrees of freedom $\mathbf{u}_{E}$. For such a reason, a stabilisation strategy similar to that used in the standard FEMs for elements with reduced integration is employed in the VEM, motivating the presence of the stabilisation matrix $\mathbf{K}_{E}^{s}$, which ensures the proper rank of the matrix $\mathbf{K}_{E}$.

The equivalent nodal forces $\mathbf{F}_{E}$ are computed as in the standard FEM from specified tractions $\overline{\mathbf{t}}$ over the element boundary $\partial E=\bigcup e_{v}$, i.e.

$$
\mathbf{F}_{E}=\int_{\partial E} \mathbf{N}^{\mathrm{T}}(\xi, \eta) \overline{\mathbf{t}} d s
$$

Once the elemental stiffness matrices and load vectors are built, the assembly of the VEM global matrices and vectors can be performed following the standard FE procedures, to obtain the following sets of equations for the VEM sub-domain

$$
\mathbf{K}^{\mathrm{V}} \mathbf{U}^{\mathrm{V}}=\mathbf{F}^{\mathrm{V}}
$$

where $\mathbf{K}^{\mathrm{V}}, \mathbf{U}^{\mathrm{V}}$ and $\mathbf{F}^{\mathrm{V}}$ are, respectively, the stiffness matrix, the nodal displacement vector and the force vector, with the superscript $\mathrm{V}$ introduced to identify quantities related with the VEM domain.

\section{Coupling procedure for BEM and VEM subdomains}

The coupling of boundary and finite elements has been achieved in the literature using various approaches [16, $17,18,19,20]$. In this work, to couple the virtual and the boundary element methods, the BEM subdomain is treated as a macro-finite element and the traction-displacement equations associated with it are transformed into force-displacement equations and assembled with the VEM equations, already expressed in terms of nodal forces and displacements.

The vectors $\mathbf{U}^{\mathrm{V}}$ and $\mathbf{F}^{\mathrm{V}}$ appearing in Eq. (11) collect the displacement and nodal force components of all the VEM nodes in the considered domain. Since only some of such nodes belong to the interface $S$, it is possible to partition the vectors as

$$
\mathbf{U}^{\mathrm{V}}=\left[\begin{array}{c}
\mathbf{U}_{S} \\
\mathbf{U}_{D}
\end{array}\right]^{\mathrm{V}}, \quad \mathbf{F}^{\mathrm{V}}=\left[\begin{array}{c}
\mathbf{F}_{S} \\
\mathbf{F}_{D}
\end{array}\right]^{\mathrm{V}}
$$

where $\mathbf{U}_{S}^{\mathrm{V}}$ and $\mathbf{F}_{S}^{\mathrm{V}}$ identify components related to nodes belonging to $S$. Along $S$, the nodal displacements and forces must then satisfy the compatibility and equilibrium conditions

$$
\mathbf{U}^{\mathrm{B}}=\mathbf{U}_{S}^{\mathrm{V}}, \quad \mathbf{F}^{\mathrm{B}}+\mathbf{F}_{S}^{\mathrm{V}}=\mathbf{0},
$$

which have been written considering that no external nodal forces act on the nodes belonging to $S$. The displacement compatibility equations can be readily written, as the same displacement components appear in both the BEM and VEM equations. On the contrary, while nodal forces appear in Eq. (11), related to the VEM domain, tractions appear in Eq. (3), related to the BEM domain, so that it is necessary to retrieve consistent nodal forces from BEM tractions, before writing the equilibrium equations appearing in Eq. (13). 
This may be done by resorting to appropriate energetic considerations, which allow to associate to the tractions acting over the considered elements a system of equivalent nodal forces $\mathbf{F}^{\mathrm{B}}$, which may be computed as

$$
\mathbf{F}^{\mathrm{B}}=\mathbf{M} \mathbf{T}^{\mathrm{B}},
$$

where $\mathbf{F}^{\mathrm{B}}, \mathbf{T}^{\mathrm{B}} \in \mathbb{R}^{2 m \times 1}$ and $\mathbf{M} \in \mathbb{R}^{2 m \times 2 m}$ is a matrix obtained by the integration of products of boundary element shape functions, with $m$ expressing the total number of boundary nodes/elements.

Eq. (3) can be written, by exploiting Eq. (14), in a form to be used in conjunction with the VEM equations; in particular, remembering that $\mathbf{T}^{\mathrm{B}}=\mathbf{G}^{-1} \mathbf{H} \mathbf{U}^{\mathrm{B}}$, it is possible to write

$$
\mathbf{F}^{\mathrm{B}}=\mathbf{M T}^{\mathrm{B}}=\left(\mathbf{M G}^{-1} \mathbf{H}\right) \mathbf{U}^{\mathrm{B}}=\mathbf{K}^{\mathrm{B}} \mathbf{U}^{\mathrm{B}} .
$$

The above BEM equations can now be combined with the VEM equations, which can be rewritten as

$$
\left[\begin{array}{ll}
\mathbf{K}_{S S} & \mathbf{K}_{S D} \\
\mathbf{K}_{D S} & \mathbf{K}_{D D}
\end{array}\right]^{\mathrm{V}}\left[\begin{array}{c}
\mathbf{U}_{S} \\
\mathbf{U}_{D}
\end{array}\right]^{\mathrm{V}}=\left[\begin{array}{c}
\mathbf{F}_{S} \\
\mathbf{F}_{D}
\end{array}\right]^{\mathrm{V}}
$$

with the interface conditions in Eq. (13) and with suitable external boundary conditions to obtain the problem solution.

\section{Crack propagation modeling using VEM}

Computational methods, such as FEM, BEM, FEM/BEM, PFEM, X-FEM, SBFEM among others, have been employed in the literature to model crack propagation processes. With finite element techniques, crack propagation is modelled explicitly by consecutively adapting the spatial discretization of the domain. The simplest method to simulate crack propagation is the nodal release technique, where a crack is extended by a certain increment along the element edge up to the next node. However, such a method restricts the crack propagation along the element edges, therefore introducing a dependence of the computed crack path on the mesh topology. More powerful but complex methods may combine element modification techniques, such element splitting, with advanced re-meshing strategies, in which the overall mesh is progressively updated starting from a finer mesh in proximity of the crack tip region. Such a continuous mesh refinement/coarsening process may generally affect large regions of the analysis domain, due to the need of preserving a conform transition between the re-meshed propagation region and the surrounding areas, and it is thus a computationally intensive operation [21].

On the other hand, VEM can handle polygonal elements of arbitrary shapes and this distinctive feature allows a substantial simplification of the re-meshing procedure following the propagation of a crack. Indeed, using VEM elements allows avoiding mesh dependency of the crack propagation direction, as any computed crack path can be represented by modifying the topology of the virtual element over which the crack propagation is occurring, including the crack edges as new element edges, without the need of further re-meshing. This straightforward approach can be employed either when the computed crack length increment is large enough to completely split an element or when it splits this element only partially. In both the aforementioned cases, the resulting element topology is a valid one from the VEM's standpoint and does not need any further mesh modification. Moreover, to improve numerical accuracy near the crack tip, a local mesh refinement can be introduced by subdividing one or more of the local elements in any number of elements of arbitrary shape, without necessarily affect large portions of the analysed domain. This can be achieved by exploiting another peculiar feature of VEM, namely its capability to naturally handle hanging nodes, which means that a VEM element can be a polygon with an arbitrary number of aligned vertices. A schematic of the modelling benefits offered by the VEM in crack propagation modelling is shown in Fig. 2.

Summarising, using VEM for crack propagation modelling allows to: $a$ ) avoid any crack path mesh-dependency, thanks to the possibility of capturing the crack propagation direction by modifying the topology of few elements in proximity of the crack tip; $b$ ) improve the accuracy of the fields reconstruction in proximity of the propagation region, thanks to the possibility of performing local mesh-refinements at low computational costs, without the need to update and optimize the mesh in large regions of the computational domain.

\section{NUMERICAL EXAMPLE}

In this section, the proposed VEM-BEM approach is applied to simulate the growth of kinked cracks originating from the partial fibre-matrix debonding in a unit cell of a fibre-reinforced composite material subjected to transverse load. 


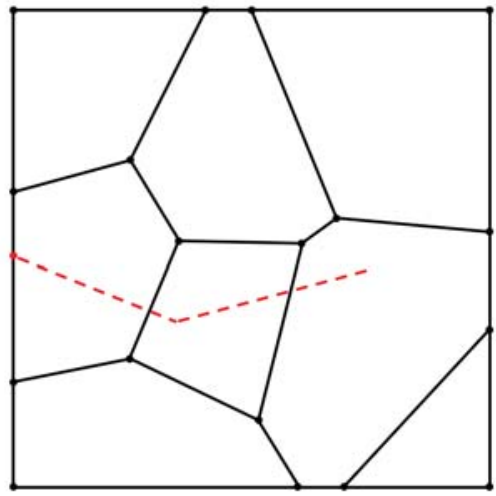

(a)

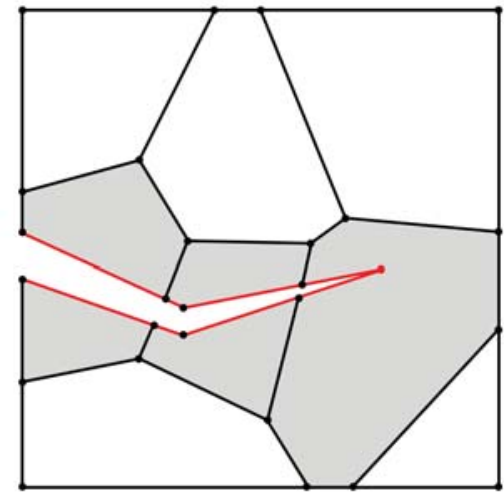

(b)

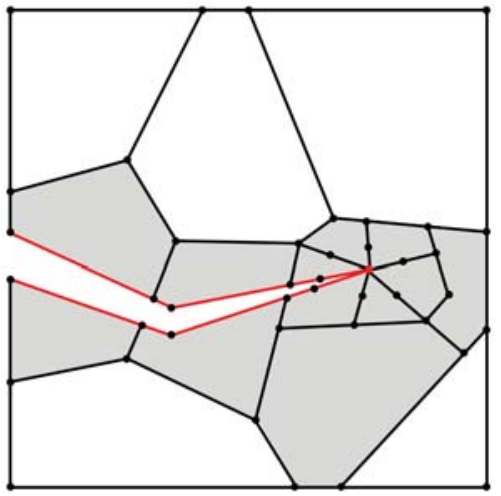

(c)

FIGURE 2: Schematic of crack propagation modelling with VEM elements: (a) computed crack path; (b) elements splitting; (c) local mesh refining.

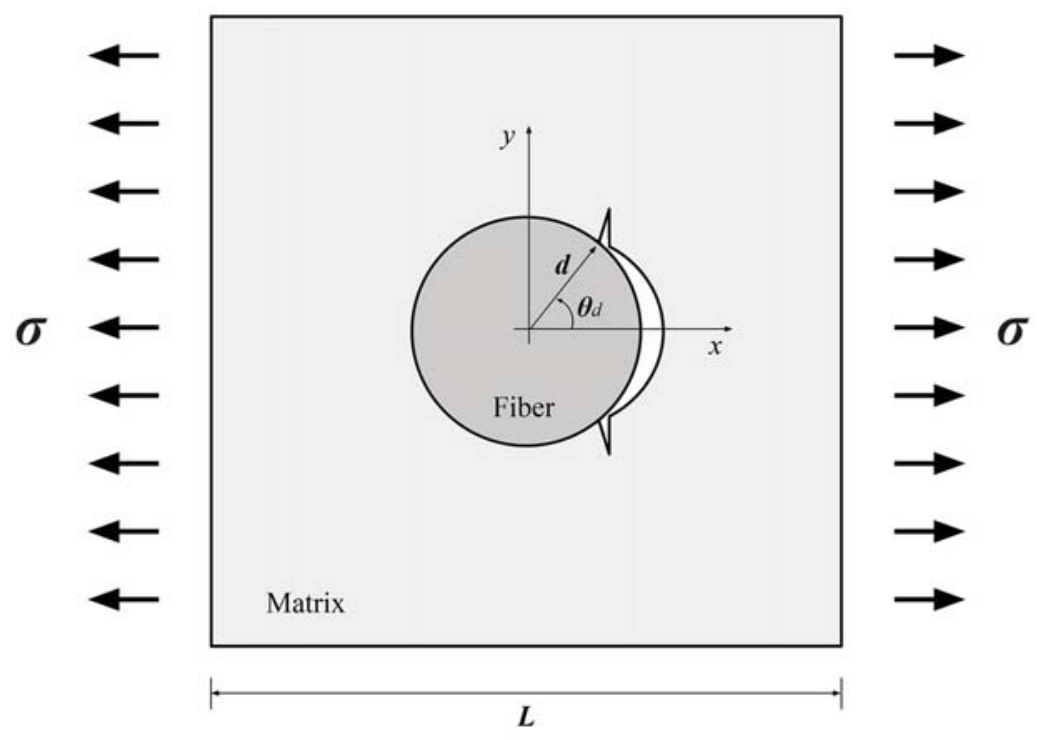

FIGURE 3: Schematic of the unit cell containing a single fiber partially debonded from the matrix.

The test case, shown in Fig. 3, represents the transverse section of a composite material unit cell consisting of a single fibre and the surrounding matrix, subjected to a tensile load $\sigma$ acting parallel to the $x$ axis. It is supposed that a crack has first grown along the interface, partially debonding the fiber from the matrix [22]. The extension of the debonded zone is identified by the angle $\theta_{d}=65^{\circ}$. Outside the debonded zone, the inclusion is perfectly bonded to the matrix. Two kinked cracks start from both ends of the debonded zone. The growth of the kinked cracks takes place only in the matrix material. The external boundary of the matrix domain is a square whose side has length $L=1 \mathrm{~mm}$. The inclusion is represented by a circle of radius $d=0.075 \mathrm{~mm}$. The center of the circle coincides with the center of the square. The initial crack length is $a=d / 5$. The initial kinked crack runs parallel to the $y$-axis. The model is symmetric with respect to the $x$-axis.

A carbon fibre/epoxy matrix composite is considered. Both the fiber and the matrix materials are treated as linear elastic under plane strain assumption. The materials of matrix and fiber, respectively, are considered as isotropic and transversely isotropic in the analysis plane. Transverse elastic properties are: Young's modulus $E_{f}=13.5 \mathrm{GPa}$ and Poisson's ratio $v_{f}=0.25$ for the fiber and $E_{m}=2.79 \mathrm{GPa}, v_{m}=0.33$ for the matrix. 


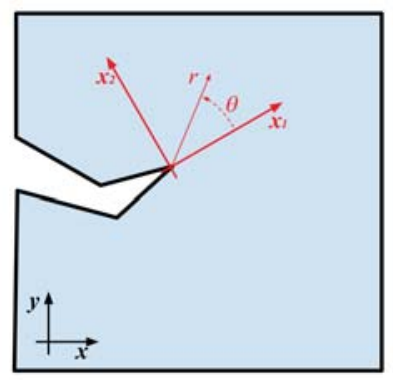

FIGURE 4: Local crack-front coordinate system.

Overall, 96 linear boundary elements are employed to model the fiber and 12034 polygonal virtual elements to model the matrix, giving a total of 48306 degrees of freedoms.

In the present study Linear-Elastic Fracture Mechanics (LEFM) is applied, i.e. geometrical and material nonlinearities are excluded. For isotropic linear-elastic material behavior the stress field near the crack tip of the mixed Mode I-II can be expressed, in polar coordinates, as [23]

$$
\begin{aligned}
& \sigma_{11}=\frac{K_{I}}{\sqrt{2 \pi r}} \cos \left(\frac{\theta}{2}\right)\left[1-\sin \left(\frac{\theta}{2}\right) \sin \left(\frac{3 \theta}{2}\right)\right]-\frac{K_{I I}}{\sqrt{2 \pi r}} \sin \left(\frac{\theta}{2}\right)\left[2-\cos \left(\frac{\theta}{2}\right) \cos \left(\frac{3 \theta}{2}\right)\right] \\
& \sigma_{22}=\frac{K_{I}}{\sqrt{2 \pi r}} \cos \left(\frac{\theta}{2}\right)\left[1+\sin \left(\frac{\theta}{2}\right) \sin \left(\frac{3 \theta}{2}\right)\right]+\frac{K_{I I}}{\sqrt{2 \pi r}}\left[\sin \left(\frac{\theta}{2}\right) \cos \left(\frac{\theta}{2}\right) \cos \left(\frac{3 \theta}{2}\right)\right] \\
& \tau_{12}=\frac{K_{I}}{\sqrt{2 \pi r}}\left[\sin \left(\frac{\theta}{2}\right) \cos \left(\frac{\theta}{2}\right) \cos \left(\frac{3 \theta}{2}\right)\right]+\frac{K_{I I}}{\sqrt{2 \pi r}} \cos \left(\frac{\theta}{2}\right)\left[1-\sin \left(\frac{\theta}{2}\right) \sin \left(\frac{3 \theta}{2}\right)\right]
\end{aligned}
$$

where $K_{I}$ and $K_{I I}$ are, respectively, the Mode I and Mode II stress intensity factors (SIF), and $r$ and $\theta$ are the coordinates of the local crack-front polar coordinate system centered at the crack tip as shown in Fig. 4.

There are several approaches for numerically evaluating the SIFs. The stress interpretation method (SIM) is one of the simplest techniques and it is based on the evaluation of the local normal stress $\sigma_{22}$ and shear stress $\tau_{12}$ on the ligament in front the crack $(\theta=0)$. From the near field solutions SIFs can be computed as:

$$
K_{I}=\lim _{r \rightarrow 0} \sigma_{22} \sqrt{2 \pi r}, \quad K_{I I}=\lim _{r \rightarrow 0} \tau_{12} \sqrt{2 \pi r}
$$

The maximum circumferential stress criterion (MCSC) [24] has been used to predict the angle $-\pi<\theta_{c}<\pi$ by which, for each crack growth increment, the new crack surface deviates from the original crack tip direction. $\theta_{c}$ is given by

$$
\theta_{c}=2 \arctan \left(\frac{1}{4} \frac{K_{I}}{K_{I I}}-\frac{1}{4} \sqrt{\left(\frac{K_{I}}{K_{I I}}\right)^{2}+8}\right)
$$

A new crack tip is added at a distance $\Delta a$ in the direction identified by the crack growth direction $\theta_{c}$. The magnitude of increment $\Delta a$ can be set arbitrarily in quasi-static loading conditions. Smaller values of the increment lead to more accurate, stable and time-consuming simulations. The assumed value is $\Delta a=d / 5$.

The computed crack propagation path for the unstable growth of the two kinked cracks is shown in Fig. 5. The obtained results agree well with previous results in [22] and, due to the relative orientation of the kinked cracks with respect to the applied load, the crack growth is dominated by Mode I.

\section{CONCLUSION}

In this contribution, we have developed a novel hybrid numerical technique combining the Boundary Element Method and the Virtual Element Method to simulate 2D linear elastic fracture problems in unidirectional fiber-reinforced 


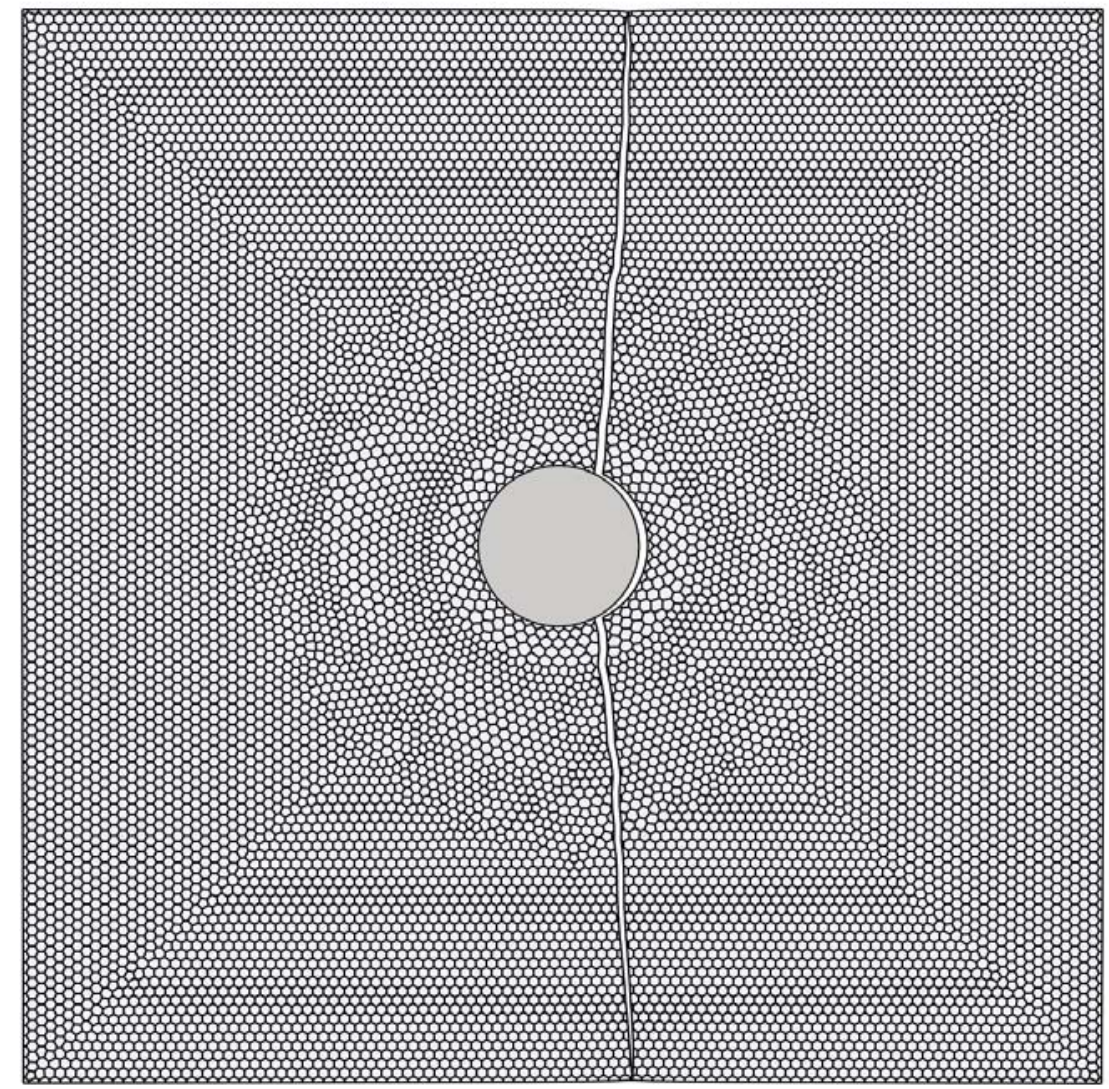

FIGURE 5: Simulated crack propagation in the considered test case.

composites. While the use of the BEM to model the fiber transverse section allows an overall reduction of the total number of degrees of freedom and a simplification of the pre-processing stage of the analysis, the use of the VEM allows a simpler treatment of the topological mesh modifications associated with crack propagation simulations. The preliminary results provided by the numerical test case show good agreement with the results found in literature and set the basis for further investigation on the accuracy, robustness and computational performance of the method.

\section{ACKNOWLEDGMENTS}

Marco Lo Cascio acknowledges the support of the Italian Ministry of Education, University and Research - MIUR - for funding the PhD scholar visit to the University of Seville, Spain. Ivano Benedetti acknowledges the support of the Italian Ministry of Education, University and Research - MIUR - through the project DEVISU, funded under the scheme PRIN-2107 - Grant 22017ZX9X4K_006.

\section{REFERENCES}

1. E. B. Tadmor and R. E. Miller, Modeling materials: continuum, atomistic and multiscale techniques (Cambridge University Press, 2011).

2. V. P. Nguyen, M. Stroeven, and L. J. Sluys, Journal of Multiscale Modelling 3, 229-270 (2011).

3. M. H. Aliabadi, The boundary element method, volume 2: applications in solids and structures, Vol. 2 (John Wiley \& Sons, 2002).

4. G. Sfantos and M. Aliabadi, Computer Methods in Applied Mechanics and Engineering 196, 1310 - 1329 (2007).

5. I. Benedetti and M. Aliabadi, Computer Methods in Applied Mechanics and Engineering 289, 429- 453 (2015).

6. I. Benedetti and M. Aliabadi, Computer Methods in Applied Mechanics and Engineering 265, 36 - 62 (2013).

7. I. Benedetti, V. Gulizzi, and V. Mallardo, International Journal of Plasticity 83, $202-224$ (2016). 
8. I. Benedetti and V. Gulizzi, International Journal of Fatigue 116, 90 - 105 (2018).

9. I. Benedetti, V. Gulizzi, and A. Milazzo, Computer Methods in Applied Mechanics and Engineering 357, 112595 (2019).

10. L. Beirão da Veiga, F. Brezzi, A. Cangiani, G. Manzini, L. D. Marini, and A. Russo, Mathematical Models and Methods in Applied Sciences 23, 199-214 (2013)

11. E. Artioli, L. Beirão da Veiga, C. Lovadina, and E. Sacco, Computational Mechanics 60, 355-377 (2017).

12. L. B. Da Veiga, A. Russo, and G. Vacca, ESAIM: Mathematical Modelling and Numerical Analysis 53, 375-404 (2019).

13. M. Lo Cascio, A. Milazzo, and I. Benedetti, Composite Structures 232, 111523 (2020).

14. M. Lo Cascio, A. Milazzo, and I. Benedetti, "Virtual element method: Micro-mechanics applications," in Key Engineering Materials, Vol. 827 (Trans Tech Publ, 2020) pp. 128-133.

15. P. K. Banerjee and R. Butterfield, Boundary element methods in engineering science (McGraw-Hill London, 1981).

16. O. Zienkiewicz, D. Kelly, and P. Bettess, International journal for numerical methods in engineering 11, 355-375 (1977).

17. C. Brebbia and P. Georgiou, Applied Mathematical Modelling 3, 212-220 (1979).

18. L. Hong-Bao, H. Guo-Ming, H. A. Mang, and P. Torzicky, Computer Methods in Applied Mechanics and Engineering 54, 161-185 (1986).

19. T. Belytschko, H. Chang, and Y. Lu, Computers \& structures 33, 17-20 (1989).

20. T. Cruse and J. Osias, "Issues in merging the finite element and boundary integral equation methods," Mathematical and Computer Modelling 15, 103-118 (1991)

21. M. Kuna, Finite elements in fracture mechanics (Springer, 2013).

22. F. París, E. Correa, and V. Mantič, Journal of Applied Mechanics 74, 703-716 (2007).

23. T. L. Anderson, Fracture Mechanics: Fundamentals and Applications, Fourth Edition (CRC Press, 2017).

24. F. Erdogan and G. C. Sih, Journal of Basic Engineering 85, 519-525 (1963). 